\title{
Über die Ehrennamen und Neubenennungen der islamischen Monate. \\ Von
}

E. Littmann.

Fast sämtliche arabischen Monate haben bekanntlich ihre Ehrennamen. Von diesen möchte ich hier, hauptsächlich um dadurch zu weiterer Forschung anzuregen, eine kurze Zusammenstellung gebes:; hierbei habe ich, da mir jetzt leider die Möglichkeit eines eingehenderenStudiums fehlt, keinerlei Vollständigkeit erstreben kónnen. Zunächst seien diese Namen in Tabellenform aufgeführt, und zwar $\mathrm{I}$. die heute in türkischen Kalendern und dergleichen gebräuchlichen Namen $\left.(T)^{x}\right)$, 2. die persischindischen Namen, wie sie mir Herr Dr. phil. des. A. Sidd IQI aus dem ihm in Indien bekannten Sprachgebrauch mitgeteilt hat (PI), 3. die Formen, die in den persischen Beischriften der Zeichnungen des Riza -i 'Abbāsī, nach der Ausgabe von SARRE und Mitrwoch (München I9I4) vorkommen (R).

I. Muharram, der Heilige.

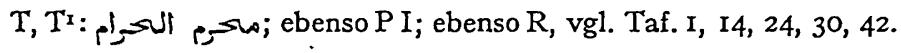

2. Safar, der Glückbringende.

T, Tx: مغر الخخير; P I: مغر المظقر; R: zweimal ohne Beinamen ختم بالخير والظغ (S. I9, Tafel I5). - Nach Tr kommt صغر المظفر in älteren türkischen Texten vor.

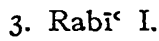

Dieser Monat heißt gewöhnlich nur ريبع الاول الاور in PI heißt er zuweilen رييع الاول النشريف, manchmal ohne Artikel beim letzten Wort.

$$
\text { 4. } \mathrm{Rabi}^{-} \text {II. }
$$

Dieser Monat hat keinen Beinamen; er heißt in $\mathrm{T}$ und PI ربيع الآخر oder ربيع آخر ohne Artikel.

1) Die im Türkischen Hilfsbuch von G. JACOB, 3. Aufl., I. Teil, S. 105 nach einer Zusarnmenstellung des Grafen E. v. MüLINEN mitgeteilten Namen sind mit Tr bezeichnet. 


\section{5. u. 6. Gumădã I und II.}

Beide Monate haben keine Beinamen. Der I. heißt in $\mathrm{T}$ regelmäßig جمانى الاونى i diese Form mag auf literarischen Purismus zurückgehen, kann sich aber auch im Bedeutungsunterschied von dem übertragen gèbrauchten جمانى الاول (s. u. S. 230 ) gehalten haben. In PI heißt dieser Monat meist جمادى الاول, da einerseits im Persischen das Gefühl für das grammatische Geschlecht verloren gegangen ist, andererseits aber auch schon arabische Vorbilder für diesc Form vorhanden sind. Daneben kommt die vom grammatischen Standpunkt aus allein richtige Form جمادى الاولى vor. R hat (S. 20) die Form mit J,ll.

Ähnlich steht es mit Gumādã II. T. hat جمانى الآخر, in der

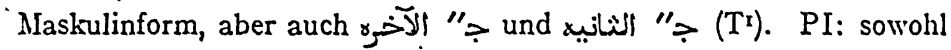

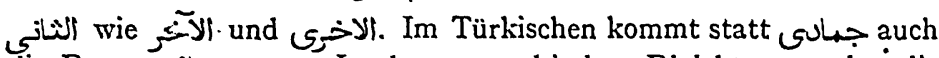
die Form جمانى vor. In den neuarabischen Dialekten werden die Zahlwörter meist in der Maskulinform gebraucht; man sagt (el-)auwel und (el-)abzar bzw. (el-)âlzir oder (et-)tânì u. ä. Dazu bildet man aber auch meist das maskuline Substantiv'gemâd, gamâd u. ä. HartmanN's Sprachführer gibt jedoch für Syrien noch die Form dschumâdi an, aber natürlich mit maskulinen Zahlwörtern.

7. Rağab, der Alleinstehende (Geschätzte).

T, Tx: رجب الفرن; PI: رجب المرجّب R (Taf. I3) wie PI.

8. Šałăn, der Verehrte.

.. T, Tr, PI u. $\ddot{R}$ (Taf. 33, 35) gleichmäßig: شعبان المعظم.

9. Ramaḍan, der Gesegnete.

T, Tx, PI u. R (Abb. 6, S. 20, Taf. I6, 47 a): :رضان المبارك; die volkstümliche Aussprache in Indien ist ramzänul-mubärak.

10. Sauwāl, der Geehrte (Glückliche).

T, T und PI: نثوال المككم; R (Abb. 5, S. I8, Taf. 2I, 28 a, 28b): ثوال با اقببل . Auf Taf. II und 20 kommt bei R شوال vor.

Ii. Dhu 1-Qa'da (der Heilige).

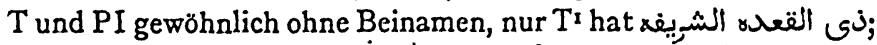

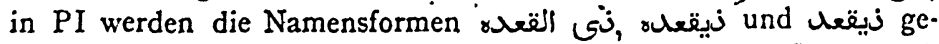
braucht. Bei R findet sich auf Taf. I2: ذيقعله المحرام

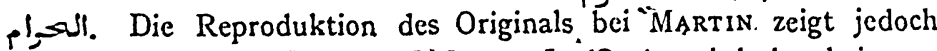

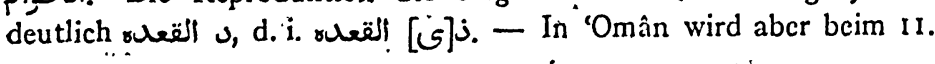


und 12. Monat das Wortchen j bzw. vj weggelassen; vgl. ReisUARDT, Ein arabischer Dialekt gesprochen in 'Omän und Zanzibar, S. 86. Ähnlich beim 12. Monat im Abessinischen; s. unten S. 235.

12. Dhu 1-Hiğge (Monat der heiligen Wallfahrt).

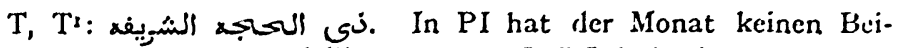

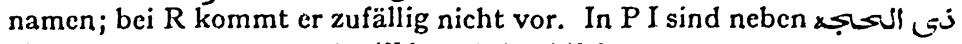
die Formen ذيحكب und zilhiğ gebräuchlich.

Zu dicsen Namen sandte mir SiddiQ1 einige Erklärungen, die ich hier mit eigenen Zusätzen wiedergebe.

I. Muharram war auch schon bei den alten Arabern vor dem Islam ein heiliger Monat, aber der eigentliche heilige Monat war der Ragab; vgl. Wellha'usen, Reste arab. Heidentums, 2. Aufl., S. 98 ff.

2. Der Monat Şafar gilt für unglücklich, und man setzt daher gern ein glückbedeutendes Wort hinzu; so im Türkischen النخير. Da aber in Muharram el-haräm eine Paronomasie vorlag und man andrerseits auch gern einen Reim hatte, so wurde Șafar el-muzaffar gewählt. Beide Ausdrücke sind bei R in ختح بالخخير والظفر vereinigt.

3. Rabic I. heißt gelegentlich التشريف, weil in ihm der Prophet geboren wurde.

4., 5., 6. haben keine Beinamen. Im Türkischen wird 5. mit maskulinem Zahlwort in übertragenem Sinne gebraucht. So gibt Kélékian s. v... الاول حمانى ,antécédents, origines " und folgende Redensarten: i je connais les origines de cette affaire; je vais vous raconter les antécédents de cet homme. Diese Redensarten haben meist eine ungun. stige Nebenbedeutung.

7. Rağab hat nach heutiger Auffassung seinen Beinamen wohl daher, weil in ihm (am 27.) Mohammeds Himmelfahrt stattfand. Wahrscheinlich geht aber die Benennung al-fard auf die alte Zeit zurück, in der dieser Monat der Hauptfestmonat war; Wellhausen, a. a. O. S. 99, weist auch mit Recht darauf hin, daß dieser Festmonat allein steht, während Muharram mit dem I1. und 12. Monat ein heiliges Quartal bildet. - Die Wahl des Wortes مرجب ist durch Paronomasie beeinflußt.

8. Der I4. Scha'bān ist der Bußtag; daher mag der Beiname stammen. Scha'bān ist übrigens auch wie Rağab und Ramaḍann ein beliebter Personenname.

9. Der Ramaḍann ist als Fastenmonat derjenige, der sich dem Bewußtsein des Orientalen am handgreiflichsten einprägt. Es ist aber 
auch deshalb "gesegnet", weil am 27. die ليلة القبر ist. Für die Wahl des Beinamens wird Süra 44× (ليلت مباركن) eingewirkt haben.

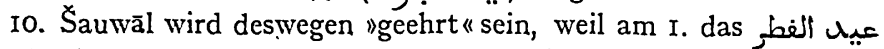

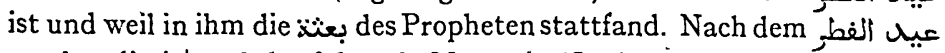
werden dieser' und der folgende Monat in 'Omân und bei muslimischen Afrikanern benannt.

II. und I2. haben gewöhnlich keine Beinamen. Das bei R für II.

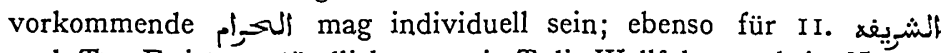
nach Tr. Es ist verständlich, wenn in $T$ die Wallfahrt auch im Namen dieses Monats als "heilig " bezeichnet wird.

Es würde sich verlohnen, der Entstehungsgeschichte dieser Beinamen näher nachzugehen. Dabei wird sich wahrscheinlich herausstellen, daß dreierlei Motive mitgewirkt haben. Zunächst sind bereits hellenistisch-ägyptische Vorbilder bekannt; zweitens wirkte das Streben nach Symmetrie innerhalb der arabischen Namen; drittens hat das Streben nach Paronomasie die Wahl einzelner Beinamen beeinflußt.

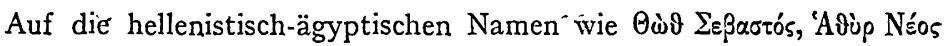

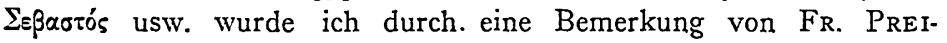
Sigke (Zum Papyrus Eitrem Nr. 5, Sitzungsber. d. Heidelb. Akad. d. Wiss. Phil.-hist. Kl. 1916, 3. Abh., S. 5) aufmerksam; er verweist dort noch auf Hohmann, ZurChronologie der Papyrusurkunden, S. 63; Gardthausen, Griech. Paläographie II, S. 474; Blumenthal, Archiv f. Papyrusforschung V; S. 344; WILCKEN, Ostraka I, S. 809. So haben wir auch hier wieder einen, wenn auch nur unwichtigen Verbindungspfad, der vom Hellenismus,zum Islam führt. — Das Streben nach Symmetrie ist in der Seele des Orientalen tief begründet; es kommt in Sprache,

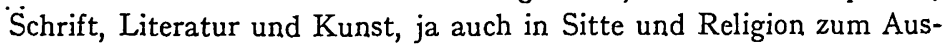
druck. Sechs Monate des arabischen Jahres bestehen aus zwei Komponenten; es war also ganz natürlich, daß.man die andern sechs Monate auch aus zwei Wörtern bestehen ließ. Ein Beweis dafür, daß dieser Gedanke mitbestimmend gewesen ist, liegt eben darin, daß jene aus zwei Wörtern zusammengesetzten Mònatsnamen für gewöhnlich keine Beinamen haben. Einen lehrreichen Fall erzählend-literarischer Symmetrie habe ich in der Festschrift für FrIEDRICH CARL ANDREAS, S. 86, angeführt. $\div$ Über die Paronomasie ist bereits S. $230 \mathrm{zu} \mathrm{Nr.} 2$ und 7 gesprochen.

Hier sei ganz kurz an die bekannte Tatsache erinnert, daß auch gewisse Städte ebenso wie die Monate ihre Ehrennamen haben: Mekka ist "die Geehrte " (المكترَّمَ); Medina 》die Glänzende". (wohl vom "Licht des Propheten ", الهنتوَّرة); Jerusalem "das Heilige " (الشيخريف); Damas- 
kus, nach Wetzstein, in ZDMG. Bd. XI, S. 501, ebenfalle ndas Heilige" (الشريف); Kerbclā "das Erhabenc" (معلى); Negef ndas Hochheilige"

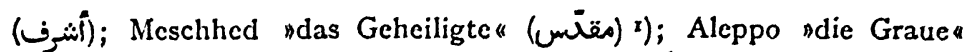
(النشيباء)); Kairo "die [von Gott] Behütete" (المحكروس)) usw. Der letztc dieser Beinamen ist aber nicht allein an Kairo gebunden; er wird auch für andere Länder und Städte gebraucht und enthält eigentlich einen 'Segenswunsch ("die von Gott behütet werden möge! (1). Auch im Altertum und im neueren Europa haben Länder und Städte ihre Beinamen bzw. Ehrennamen. Da gibt es "Roma Eterna ", "Napoli la Bella", "Merry Old. England " und "la gaie Gaule", sogar das "Heilige Rußland".

Im Zusammenhang mit den Ehrennamen teilte mir Siddror aber auch die in Indien volkstümlichen und in der Frauensprache allgemein gebräuchlichen Namen der islamischen Monate mit. Sie lauten: I. Möhørram, Møhram, Mhöram (je nach der Gegend). -

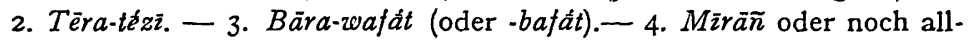
gemeiner Dastgír, Pìr(-e) dastgìr. - 5. Madăr. - 6. Hamsáya, Husénšavalì (d. i. Husain-šāh-valī). - 7. Rağab, Rağğab. - 8. Sabbarát (oder Subrát) < sab-e barät. - 9. Ramzán oder lieber Rōzē (Plur. von pers.

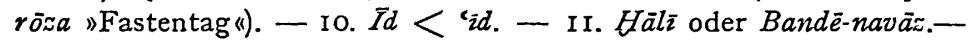
12. Bakrïd, Baqrïd < baqar-ìid; Td-c qurbān.

Zur Erklärung schrieb mir SidDiQI folgendes:

"2. Tèra-tēzì. Têra bedeutet "dreizehn " "scharf ". Der Name bedeutet wahrscheinlich "der mit den I3 schweren Tagen «. Der Monat gilt für unglückbringend, die ersten 13 Tage gelten für besonders unheilvoll, und der 13 . ist wieder unter diesen I3 Tagen der schlimmste Tag. - Die I3 als Unglückszahl ist ja auch anderswo weit verbreitet.

3. Bära heißt "Zwölf", wafät ist das arabische وفات. Der I2. $\mathrm{Rabi}^{\prime} \mathrm{I}$. ist der Geburtstag wie auch der Todestag des Propheten.

4. In diesem Monat ist der Todestag eines Walĩ namens Mĩräñ ; der Name Mīrāan bzw. Mīrān kommt oft vor. Der II. ist der Todestag des 'Abd el-Qādir el-Gīlānī, der in Baghdād begraben ist und der für den größten Walī gehalten wird. Er hat sehr viele Beinamen: I. حفرت

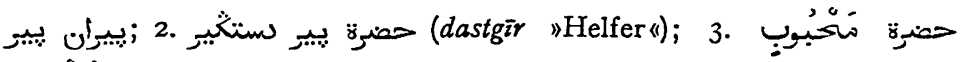
سُبْْ Doner. Daher der Moname.

1) An die Beinamen von Kerbelā, Neğef und Meschhed erinnerte mich Swdiqr; مشتهح sقدس kommt auch bei Rizã-i 'Abbāsĩ, Tafel r, vor. 
5. Madär ist wiederum ein Walī ( $\mathrm{P} \overline{\mathrm{r}} \mathrm{r})$. In andern Gegenden wird der Monat auch nach andern Walis benannt.

6. Den Nameñ Hamsäya (pers.) "Nachbar» kann ich nicht erklären. Husain Šăh ist wieder ein Walī; so wird der Monat im Dakan genannt.

8. Sab-e barät "die Nacht der Feier " ist"die I4. Nacht des Monats. Das ist etwas besonders Schönes für die Kinder: überall ist Beleuchtung und Feuerwerk, zum Essen gibt es Halwa, weil der Prophet in der

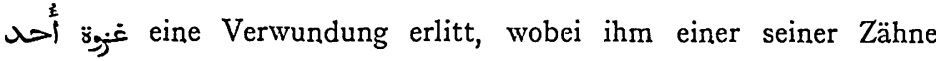
ausfiel. Er konnte also nichts Hartes beißen und aß deshalb Halwa. In Wirklichkeit ist dieser Tag etwa wie der Totensonntag in christlichen Ländern. Jede Familie betet in dieser Nacht für ihre Toten, und viele eßßbare Dinge werden unter die Armen verteilt. 'Die Beleuchtung und das Feuerwerk werden erklärlich, wenn man an eine ähnliche Feier der Hindus, namens Divälï, denkt. Die muslimischen Geistlichen haben dafür gesorgt, daß die Kinder ihrer Gemeinden nicht gemeinschaftlich mit den Heidenkindern die Divālī zu feeiern brauchten.

I0. $I d$ "Fest « wird der Monat nach dem عيد الفحر genannt. [Den zweiten Teil des Namens dieses Festes haben die Südaraber und Afrikanèr gewählt; s. u. S. 235.]

II. Hâli "leer" heißt dieser Monat, weil in ihm kein Fest stattfindet, während der vorhergehende und der, nachfolgende Monat Festmonate sind. Aber im Dakan gab es einen Wali, dessen 'Urs am I7. dieses Monats stattfindet; er hatte den Beinamen "Bandē-navāz.

I2. Im Hindustani heißt bakra'»Ziegenbock», bakrī "Ziege». Weil am I0., II. und $і 2$. des Monats Ziegenböcke geschlachtet werden, nennt man das Fest "Ziegenfest". Die Form baqrìd ist so zu erklären, daß den Gebildeten das echt indische Wort nicht lieb war; sie haben deshalb, nur um ein arabisches Wort gebrauchen zu können, ' baqar-' $\bar{\imath} d(>$ baqrī $d)$ gesagt und die Ziege zum Rinde gemacht."

Eine sehr ausführliche Beschreibung der indischen Monate und Feste findet sich bei HerkLots, Qanoon-e-Islam, Madras, 1863, S. $98 \mathrm{ff}$. Auf dies wichtige Kapitel machte mich C. H. BECKER freundlichst aufmerksam; auch erinnerte er mich an die von SNoucx Hurgronje in seinem Buche, The Ashehnese I, S. I94 f. zusammengestellten und erklärten Monatsnamen sowie an den Artikel von E. GraEFE im Islam IV, S. I6r.

Zu den indischen Namen bietet die türkische Frauensprache einige schöne Parallelen; diese Monatsnamen der Frauen sind nach Graf E. voN MÜLINEN in der von G. JACOB herausgegebenen Türkischen 
Bibliothek, 13. Band, S. 106, mitgeteilt. Dort finden sich an Neubenennungen: "1. 'Aschâre für muharrem; 3. büjük mevlad (wegen der Gcburt des Prophcten) für rebı゙ ul-evvel; 4. kütschük mevlad (in Analogic des Vorhergehenden) ${ }^{2}$ ) für rebí us-sâni; 5. büjük tevbe; 6. kütschük tevbe (für dic beiden dschemázy); 10. bairam, weil mit diescm Feste der schevval beginnt; I1. aralyk aji (zwischen den beiden Bairams) für $z i$ 'l-qa'de; 12. qurbân für zi' $l$-hiddsche.« - Dazu kommen noch nach MenzeL, Islam IV, S. I26; 3. Ilk mevlud; 4. Son mevlud'); 7. u.8. Namaz ajlary und Io. Bairam ajy.

Überall haben Namen der Feste den Anlab zu neuen Benennungen gegeben. Es sei noch bemerkt, daß Kélékian im Wörter-

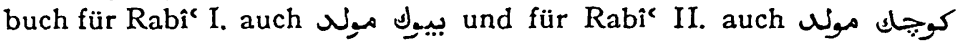
als volkstümliche Namen gibt. Die Form mevlâd (so voN MüLINEN und MENZEL) ist wohl gebräuchlicher als das literarisch klingende mevlid (so Kélékian) 2).

Ganz ähnlich wie in Indien und bei den türkischen Frauen hat sich die Benennung der Monate in Nordabessinien bei den christlichen Tigrè-Stämmen entwickelt. Dort sind die altäthiopischen Namen fast ganz geschwunden; nur Mai und Juli haben die alten Namen behalten, für Dezember kommt der alte Name noch vor. Die Namen sind folgende (vgl. meine Publications of the Princeton Expedition to Abyssinia II, S. 67 f.): I. September: Yallannes (nach dem Johannes-Fest). - 2. Oktober: Masqal "Kreuz" (nach dem Kreuzauffindungsfeste, dem 2. großen Feste im Jahre, das aber noch in den September fällt). 3. November: Mekke'ēl-qaim "Herbst-Michael «. - 4. Dezember: Gabre'èl oder Tahasās (das alte Tälısiäs in gemischter amharischer und tigrischer Aussprache). - 5. Januar: Astar'iyo "Epiphanias «. - 6. Februar: Șôm "Fasten ". - 7. März: Keflā "seine Hälfte", vgl. den deutschen Tagesnamen "Mittwoch "statt des alten Wodantages sowie oben Z. 6 aralyk aji. - 8. April: Fäzagā "Ostern ". - 9. Mai: Gembōt. - ro. Juni: Mekke'êl-hagäy "Sommer-Michael ". - I I. Juli: Hamlē. - I2. August: Mãryäm "Maria «; denn in diesem Monate findet das größte der vielen Marienfeste statt.

Solche Benennungen der Monate nach Festen kommen schon im alten Ägypten und Babylonien vor. Sie werden sich auch in anderen islamischen. Ländern nachweisen lassen; vgl. Islam IV, S. 16I.

Merkwürdig haben sich die islamischen Monatsnamen bei den mohammedanischen Tigrē-Stämmen entwickelt. Sie haben die beiden

$\left.{ }^{2}\right)$ Im Rabi' II. wird der Geburtstag des Husain gefeiert.

2) Nach SIDDIQI ist maulūud auch in Indien die gebräuchliche Form, während maulid dort nur in Buchern vorkommt.! 
Jahreshälften miteinander vertauscht und fangen also das Jahr mit dem Rağab an; es ist möglich, daß sich hierin eine alte Tradition erhalten hat. Wir haben nun folgende Entsprechungen:

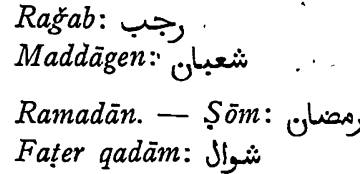

Faṭer lıăr: نو القعده:

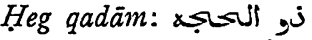

Heg liār. - Ma'ašúrāa:

Im Tigrē bedeutet qadām "erster", hăar "letzter ". Hier sind sogar, aus praktischen Bedürfnissen, wie wir gleich sehen werden, die beiden Monate مالكي zu Monaten mit igleichen Namen zusammengefaßt, und dadurch ist die Teilung des Jahres in zwei Hälften, die im Namen Keflä (bei den Christen) noch so deutlich hervortritt, ganz unkenntlich geworden. Die Zusammenfassung der beiden Monate findet sich aber auch in den von E. GRAefe a. a. O. mitgeteilten Namen, Ed-Dahië el-evvele und Ed-Dahië et-tanië.

Maddāgen bedeutet "Verfolger", also hier der auf den I. Monat folgende Monat. - Dies ist ein neuer Bewcis, wenn es eines solchen überhaupt bedarf, für die Richtigkeit der Erklärung des Namens سائق الك_أمات durch C. H. Becker, vgl. Islam IV, S. I6I.

Șōm, der andere Name für Ramadän, bedeutet "Fasten «; dazu vergleiche man die volkstümliche indische Benennung des Monats. Fater I. ist nach dem ' $\bar{I} d$ el-Fițr am I. Sauwāl so benannt; die Inder brauchen hier nur $\bar{I} d$. Um ein Paar zu gewinnen, nannte man den

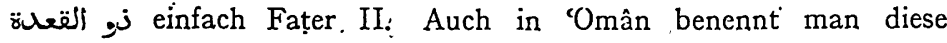
beiden Monate ähnlich, man sagt fatri l-auli und fatri l-ahîr, beide werden auch unter den Namen l-fatriyāt oder $\xi$-sewäwīl zusammengefaßt; vgl. Reinhardt, a.a. O. S. 87. E. Graefe führt aus Afrika die Namen El-fitr el-evvel und El-fitr et-tani an. - In Heg I. erkennt

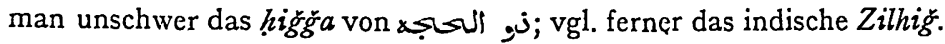
Der nächste Monat, dessen Bedeutung man in Abessinien gänzlich vergessen hatte, wurde $\mathrm{zu} \mathrm{Heg}$ II. umgetauft oder, wie in der tür-

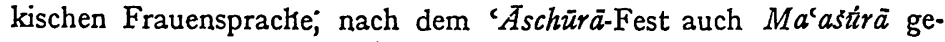
nannt. - Die folgenden fünf Monate haben ihre arabischen Namen beibehalten; nur sind die Formen im Tigrë ètwas verändert.

$\mathrm{Zu}$ den Monaten wurde mir folgendes berichtet: "In den Monaten Ray̆ab, Maddāgen, Ramạdān und Safar heiratet runḍ verheiratet man nicht. Aber wenn Leute große Eile haben, so kann einer, der keine 
Brüder hat, oder auch eine, die keinc Brüder hat, in ihnen heiraten. Dic abcr, die Brider haben, heiraten in ihnen nicht. Vielmehr heiratet man immer in denjbeiden Fater ${ }^{\mathrm{y}}$ ), den beiden $\mathrm{Heg}$, den beiden Rabe und den beiden Gemad."

Diese ganze Sitte beruht auf Aberglauben: Heirat ist die Vereinigung zwcier Menschen, und die darf es nur in Monaten geben, deren es auch zwei gibt; sonst könnte einer der beiden Heiratenden bald wieder so einsam werden wie der Monat, in dem man geheiratet hätte. Aber "wenn man große Eile hat ", kann man auch in den Einzelmonaten heiraten; nur darf man keine Geschwister haben, da die Einsamkeit des Heiratsmonats den Geschwistern Unglück bringen könnte. Wir haben hier also Symmetrie in Glauben und Sitte. Diese Sitte ist aber natürlich ziemlich jung. Zunächst nahm man die islamischen Monatsnamen mit ihren zwei Doppelmonaten herüber. Dann kam die Sitte des Heiratens in diesen Monaten auf, und man brauchte allmählich mehr Doppelmonate; so wurden aus zweien deren vier. Dabei konnte man sich wahrscheinlich an Vorbilder bei Südarabern und bei afrikanischen Muslimen anlehnen. Und in Religion und Aberglaube muß man die Bedürfnisse des praktischen Lebens nicht außer Augen lassen; so gelten z. B. auch alle die Vorschriften wegen Unglückshaare bei den Rindern nur für die Bullkälber. Wenn bei ihnen die Unglückshaare gefunden werden, so werden sie geschlachtet; Kuhkälber sind aber zu wertvoll, um geschlachtet $\mathrm{zu}$ werden, ihre Unglückshaare gelten darum nicht.

x) Nach ReinhardT, a. a. O. S. 87 , sind jedoch die beiden Šauwāl (d. i. కanuwāal und $l$ - $\left.q a^{2} a d e\right)>$ fur die Verehelichung ungluckbringende. 